\title{
ReMed: rapport annuel 2013/2014
}

Comité de direction de ReMed

\section{ReMed Suisse}

A l'instar de l'année dernière, ReMed a pu continuer à accroître sa notoriété et à sensibiliser les médecins au réseau de soutien en participant à des congrès (notamment à Montreux et à Dublin avec des organisations internationales apparentées) et à des ateliers. Cet ancrage plus marqué se reflète également dans le nombre d'appels, qui a subi une légère hausse en 2013. Les prestations de ReMed sont toujours plus sollicitées d'année en année. La plupart des appels portent sur des problèmes de burnout et de dépression, suivis par des problèmes de gestion du cabinet et du quotidien. Il est frappant de constater que les médecins travaillant en institution ont été particulièrement nombreux à faire appel à ReMed.

\section{Mise en réseau renforcée}

Afin de pouvoir proposer un soutien professionnel aux personnes qui s'adressent à ReMed, le Comité de direction organise depuis 2009 des rencontres d'intervision pour les médecins de Suisse romande et de Suisse alémanique. Les confrères qui y participent proposent aux médecins qui ont recours à ReMed du coaching, des conseils ou une thérapie selon les besoins de la personne concernée. Notons que le nombre de rencontres et de participants s'est stabilisé au cours de l'année passée sous revue. Lors de ces rencontres d'intervision, de nombreuses questions tirées de la pratique ont été abordées: crises personnelles ou familiales de confrères, crises liées à des transitions de vie (passage de l'hôpital en pratique privée, remise ou reprise de cabinets), fautes médicales, abus, problèmes d'addiction, burnout, questions juridiques ou d'assurance, conflits institutionnels ou hiérarchiques dans les hôpitaux ou les cabinets (de groupe). La mise en réseau sera poursuivie cette année, en y incluant également le Tessin. Toute personne intéressée à collaborer avec ReMed est la bienvenue.

\section{Augmenter la notoriété}

En 2013 également, les médias ont publié de nombreux articles sur ReMed et plusieurs témoignages de personnes ayant bénéficié du soutien de ce dernier ont été publiés dans le Bulletin des médecins suisses. Ces témoignages suscitent toujours un vif intérêt auprès des lecteurs et font l'objet de retours très posi- tifs. En janvier 2014, des dépliants ReMed ont été envoyés aux responsables qualité de tous les hôpitaux de Suisse en les invitant à bien vouloir les diffuser dans leur institution.

\section{Sensibilisation des médecins}

En février 2013, une nouvelle représentation interactive du théâtre Knotenpunkt sur le thème du burnout chez les médecins a eu lieu à Sargans. Le succès rencontré montre bien l'intérêt de ces manifestations pour faire connaître ReMed aux médecins intéressés. Le Comité de direction se penche actuellement sur la manière d'intégrer de telles représentations dans les programmes de formation continue. Une troupe de théâtre francophone a été approchée et le projet devrait voir le jour en Suisse romande dans le courant de l'année 2014 déjà.

\section{ReMed - changement au sein de la direction du programme}

En juin 2013, Esther Kraft, cheffe de la division Données, démographie et qualité de la $\mathrm{FMH}$, a repris la direction stratégique du programme en collaboration avec Michael Peltenburg, responsable du programme. Composé de sept membres, le Comité de direction de ReMed est responsable de l'organisation et du développement de l'offre de soutien. Suite à la hausse du nombre de demandes, il devra une nouvelle fois être élargi au cours des années à venir. A l'instar de l'année dernière, les organisations consultatives de ReMed ont été invitées à participer aux séances du Comité de direction afin de favoriser l'échange d'informations et de renforcer les contacts existants. Par ailleurs, des rencontres ont régulièrement eu lieu avec Christine Romann, membre du Comité central de la FMH et responsable du département Prévention et promotion de la santé, et Barbara Weil, cheffe de la division Prévention et promotion de la santé. Dans le but de consolider son rôle dans le domaine de la «violation du respect de la dignité humaine», le Comité de direction a reçu deux déléguées du groupe de travail éponyme. Pour clarifier les questions juridiques, il a également convié Hanspeter Kuhn, avocat et chef de la division Service juridique de la FMH. En 2014, le Comité prévoit d'encourager et de poursuivre les échanges avec les représentants des organisations consultatives. 